\title{
A Polar Coordinate System Based Grid Algorithm for Star Identification
}

\author{
Hua ZHANG ${ }^{1}$, Hongshi SANG ${ }^{*}$, Xubang SHEN ${ }^{2}$ \\ National Key Laboratory of Science and Technology on Multispectral Information Processing technologies, Huazhong University of \\ Science and Technology, Wuhan, China; ${ }^{2}$ Xi' an Microelectronics Technology Institute, Xi'an, China. \\ Email: hankz007@163.com, sanghs@mail.hust.edu.cn
}

Received September $3^{\text {rd }}, 2009$; revised September $28^{\text {th }}, 2009$; accepted October $6^{\text {th }}, 2009$.

\begin{abstract}
In Cartesian coordinate systems, the angular separation-based star identification algorithms involve much trigonometric function computing. That delays the algorithm process. As in a polar coordinate system, the coordinates are denoted by angular values, it is potential to speed up the star identification process by adopting a polar coordinate system. An angular polar coordinate system is introduced and a grid algorithm based on the coordinate system is proposed to enhance the performances of the star identification process. The simulations demonstrate that the algorithm in the angular polar coordinate system is superior to the grid algorithm in the rectangle Cartesian coordinate system in computing cost and identification rate. It can be used in the star sensors for high precision and high reliability in spacecraft navigation.
\end{abstract}

Keywords: Star Identification, Grid Algorithm, Polar Coordinate System, Star Sensor

\section{Introduction}

As the star sensors are used widely in autonomous spacecraft navigation, the star identification algorithm must be of time efficiency and high identification rate. Most star identification algorithms employ the angular star pair distance or polygon to build the guide database. These algorithms include polygon match algorithm, triangle algorithm [1-4] and group match algorithm [5-7], etc. Another class of star identification algorithms accomplishes star identification in terms of pattern recognition or best match. These algorithms associate each star with a pattern or signature determined by its surrounding star field. Then the star identification process can be treated as finding the closest match between the observed patterns and the catalog patterns. The most representative algorithm in this class is grid algorithm [8]. Compared with other algorithms, grid algorithm is an excellent algorithm with a higher identification rate and smaller memory requirement, as well as it is computationally efficient [9]. However, this algorithm needs to find the closest neighboring star of the reference star to generate a star pattern. As many fixed stars are variable in visual brightness and the measuring noises exist in measurement, the identification probability for the closest neighboring star is comparatively low. That leads to identification failure resulting from misidentifications.
The literature [10] proposes a new grid algorithm adopting the radial and cyclic features of the stars in star identification. The algorithm demonstrates excellent performance in identification rate.

Recently, almost all the star identification algorithms are based on the Cartesian coordinate system. Imperfectly, the angular separations must be computed by using trigonometric functions. That delays the star identification process. As is known, the polar coordinate system involves the angular information in the coordinates. It is potential to improve the star identification algorithm in the round polar coordinate system. In this paper, an angular polar coordinate system is proposed for the star sensor and a grid algorithm in the proposed polar coordinate system is introduced. In the simulations, the algorithm proposed is compared with the grid algorithm in the Cartesian coordinate system introduced in the literature [10]. As the simulations demonstrate, the algorithm in the angular polar coordinate system is superior to that in the Cartesian coordinate system.

The paper is separated into four major sections as follows: the angular polar coordinate system for the star sensor is introduced in the second section, and the grid algorithm for star identification in the angular polar coordinate system is introduced in the third section. In the last two sections, the simulations are presented and the results are discussed. 


\section{Angular Polar Coordinate System for Star Sensor}

The polar coordinate system is well known and used widely. The proposed coordinate system is derived from the conventional polar coordinate system to be used in focal length related image processing field, in that the radial coordinate is denoted by an angular value, so named angular polar coordinate system. As shown in Figure 1, the radial coordinate is denoted by $\varphi$, which is the view angle from the focus of the lens physically. And the angular coordinate is denoted by $\theta$, just the same as the angular coordinate in the conventional polar coordinate system [11]. The coordinate angles in the polar notation are expressed in either degrees or radians $(2 \pi \mathrm{rad}$ being equal to $360^{\circ}$ ), and the angular coordinate $\theta$ is measured counterclockwise from the axis and limited to be non-negative values. The axis for $\theta=0$ is chosen to be the same as the direction of the $\mathrm{X}$ axis of the image sensor in the Cartesian coordinate system. Then the two polar coordinates $\varphi$ and $\theta$ can be converted to the Cartesian coordinates $\mathrm{x}$ and $\mathrm{y}$ by using the trigonometric functions,

$$
\left\{\begin{array}{l}
x=f \tan \varphi \cos \theta \\
y=f \tan \varphi \sin \theta
\end{array}\right.
$$

where $f$ is the focal length of the sensor lens.

In the Cartesian coordinate system, if the coordinates of the two stars in the planar frame of the star sensor are $\left(x_{i}, y_{i}\right)$ and $\left(x_{j}, y_{j}\right)$, the two unit vectors below can denote the stars in the body coordinate of the star sensor,

$$
v_{i}=\frac{1}{\sqrt{x_{i}^{2}+y_{i}^{2}+f^{2}}}\left[\begin{array}{c}
x_{i} \\
y_{i} \\
-f
\end{array}\right], \quad v_{j}=\frac{1}{\sqrt{x_{j}^{2}+y_{j}^{2}+f^{2}}}\left[\begin{array}{c}
x_{j} \\
y_{j} \\
-f
\end{array}\right]
$$

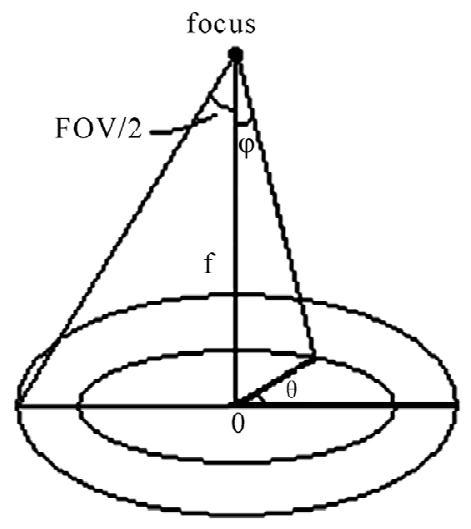

where $f$ denotes the focal length of the optical lens in the star sensor. Then the angle between the two vectors can be computed,

$$
e_{i j}=\arccos \left(v_{i} \bullet v_{j}\right)
$$

In star identification process, the two stars are called a star pair, and the angle between the two stars is called the angular separation or angular distance between the star pair. The principle of angular separation matching is that the angular separations between the star pairs in the star image are computed and compared with those stored in the guiding catalog. If the differences is within an error bound on the expected errors, the angular separation pattern is considered matched and the stars made up of the star pair can be determined.

While in the angular polar coordinate system, if one star is located at the polar point, the radial coordinate of another star is just the angular separation between the two stars. And the angular coordinate can represent the distribution of the star relatively, as shown in Figure 2. Therefore, it is potential to use the angular polar coordinate system for star identification to avoid computing the angular separations.

In the polar coordinate system, the polar grids are used. As shown in Figure 2, the planar image frame is divided into mean radial and cyclic angular grids. In both coordinate directions, the frame can be divided by mean angular distance. The distribution of the stars in the frame can be denoted by the angular grids. We use the concepts of the radial and cyclic feature in the literature [10] and realize a grid algorithm for star identification in the angular polar coordinate system to improve the performance of the algorithm.

\section{Star Identification Algorithm in the Polar Coordinate System}

The concept of the algorithm we propose is to implement fast coarse star identification by using the distribution of the observed stars in the FOV (field of view) of the star-

Figure 1. Angular polar coordinate system for star sensor 


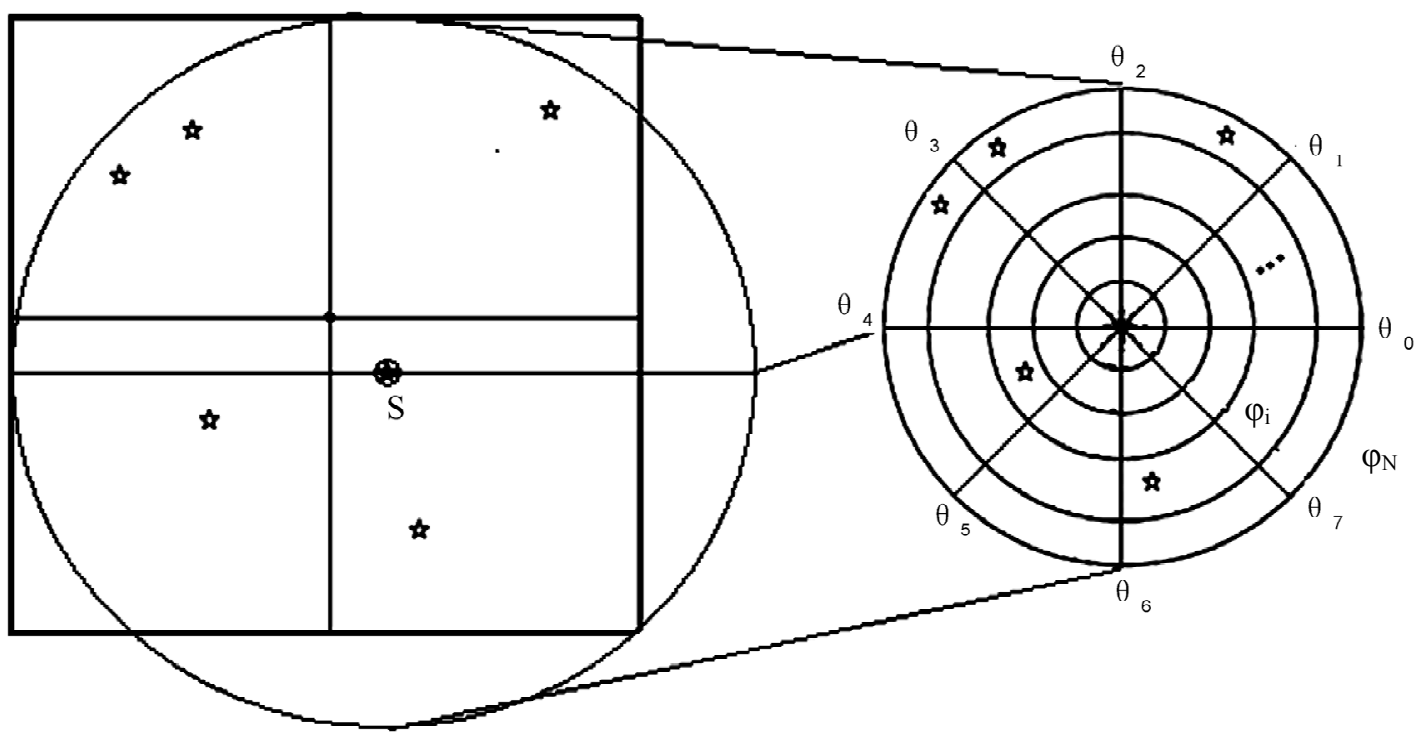

Figure 2. The round grid in the polar system

sensor, without the need for computing the angular separations. The algorithm involves three steps: first, an angular polar coordinate system is built to denote the locations of the stars, and second, the star patterns are generated in the angular polar coordinate system. Then the coarse star pattern matching is performed and the accurate star identification is realized by checking the angular separations between the stars.

\subsection{Angular Polar Coordinate Frame Building}

As the locations of the stars captured in the FOV are extracted, the brightest star S near the center of the FOV is chosen as a reference star. The other stars around the reference star $\mathrm{S}$ are called the neighbor stars of $\mathrm{S}$. An angular polar coordinate system is generated with the polar point at the center of the reference star $\mathrm{S}$ and the polar axis same as the direction of the $\mathrm{X}$ axis of the image sensor in the Cartesian coordinate system. To avoid computing the trigonometric functions in Equation 1, a lookup Table (LT) is used to map the coordinates of the stars in the planar image frame to the angular polar coordinate system.

A round neighbor area of the reference star $\mathrm{S}$ is generated in the new coordinate system, as shown in Figure 2, with the radius of the round area covering all the stars in the FOV of the star sensor. Then the polar grids are partitioned by mean radial angles and mean angular distance. To reduce the redundancy of the computing cost, the radial pattern and the angular pattern are generated separately.

\subsection{Star Pttern Gneration}

\subsubsection{Radial Feature Pattern}

Evenly partition the round neighbor area of the star S into $\mathrm{N}$ ring strips, where each ring strip represents an angular distance of 0.01 degree. As for the sensor pattern, the number of $\mathrm{N}$ is determined by the location of the star $\mathrm{S}$ in the star image, equal to the largest number of ring strip where there is at least one star within the ring strip. Then the radial pattern of the star $\mathrm{S}$, pat $t_{r}(S)$, is determined by

$$
\operatorname{pat}_{r}(S)=\left(B_{1}, B_{2}, \ldots, B_{N}\right)
$$

where

$$
B_{i}=\left\{\begin{array}{l}
1, \text { if there is at least a star between the radial grid } \varphi_{\mathrm{i}-1} \text { and } \varphi_{\mathrm{i}} ; \\
0, \text { else. }
\end{array}\right.
$$

The catalog radial patterns are generated just as the process of generating the sensor radial pattern. To compatible with the unusual conditions that the reference star is near the brim of the FOV, the number of $\mathrm{N}$ in generating the catalog radial patterns is so set that the radius of the round area is twice the size of the FOV, i.e. $\mathrm{N}=100 \times \theta$ where $\theta$ is the angle of the FOV in degree. Therefore the number of the bits in a catalog radial pattern is larger than that in a sensor radial pattern. Accordingly, in the radial pattern matching process, when the numbers of the catalog radial pattern is longer than that of the sensor radial pattern, the extra bits are neglected.

To reduce the memory requirements of the star catalog, the radial patterns can be expressed by numbers of bytes 
where every $B_{i}$ is denoted as a bit.

\subsubsection{Angular Feature Pattern}

Evenly partition the round neighbor area of $S$ into 8 angular sectors, with each sector representing an angular distance of 45 degree in the angular direction, as shown in Figure 2. A vector $v=\left(A_{1}, A_{2}, \ldots, A_{8}\right)$ is obtained from the neighbor stars' distribution in the angular sectors in an anticlockwise direction, where

$$
A_{i}=\left\{\begin{array}{l}
1, \text { if there is at least one star between the angle grid } \theta_{\mathrm{i}-1} \text { and } \theta_{\mathrm{i}} \\
0, \text { else. }
\end{array}\right.
$$

When two or more stars lie in the same sector, the corresponding bit of this sector is set to 1 just like the situation that only one star lies in the sector. Shift $v$ to the left circularly to find the maximum byte formed by $v$ where each $A_{i}$ is treated as a bit sequentially. As a result, the maximum byte is defined as the angular pattern of the star S. For instances, when there is only one star within the round area, the angular pattern of the star, pat $_{a}(S)=128$. Especially when there is no neighbor star within the round neighbor, $\operatorname{pat}_{a}(S)=0$.

The catalog angular patterns are generated just as the process of generating the sensor angular patterns described above. However, the radius of the round area around the reference star is twice of the size of the FOV of star sensor.

\subsection{Star Pattern Matching}

In the pattern matching process, a counter is used to represent the likeness between the observed sensor patterns and the catalog patterns. For every catalog radial pattern, if any $B_{i}=1$ both in the sensor pattern and in the catalog pattern, the likeness counter plus one. The counter remains invariable whenever $B_{i}=0$ or the responding $B_{i}$ in the sensor pattern is not as same as that in the catalog pattern. The star with the highest counter value is considered as the candidate star. In the same way, for every angular pattern in the catalog, if any bit equals to one both in the sensor angular pattern and in the catalog angular pattern, the likeness counter plus one. As the angular distribution of the neighbor stars is invariant of rotation, the sensor angular pattern is shifted circularly till a maximal likeness counter value is gotten. And the star with the highest counter value is considered as the coarsely-matched star. Then the angular separations among the stars are computed to make sure exactly which star is in the FOV.

In short, the star identification algorithm proposed can be described as below:

1) Choose a brightest star $\mathrm{S}$ near the center of the FOV as the reference star and the polar point, then an angular polar coordinate frame is built around the reference star $\mathrm{S}$.
2) A round area around the star S is generated and partitioned into $\mathrm{N}$ ring strips and 8 angular sectors.

3) The sensor radial pattern is generated and matched with the patterns in the star catalog. If there is only one candidate star, the coarse identification process is complete. The candidate star is considered as the coarselymatched star, and the algorithm goes to step (5). On the contrary, if there are more than one candidate star, the following step are carried out.

4) For each candidate star, the angular pattern is generated and matched with the catalog angular patterns. The star with the highest likeness counter value is considered as the coarsely-matched star.

5) If there is only one coarsely-matched star, the angular separations between the neighbor stars and the reference star is computed and checked with the angular separations in the catalog to distinguish each star. If there is more than one coarsely-matched star, the angular separations among the neighbor stars are computed to eliminate the false coarse matches. Then the angular separations between the neighbor stars and the reference star is computed and checked to distinguish each star.

\section{The Simulations and Discusses}

The algorithm proposed has proven successful in night sky tests. The star sensor used for the experiments is a prototype star sensor, which uses STAR250, a $512 \times 512$ pixels APS (active-pixel sensor) image sensor manufactured by Cypress (Fillfactory). To evaluate the algorithm presented in the paper, Monte-Carlo simulations are performed on the virtual photos taken on the full celestial sphere with various noises inserted into the centroids of the stars in the virtual star images. The star sensor configuration used for the simulations makes use of an $18 \times 18$ degree FOV with an image resolution of $1024 \times$ 1024 pixels. The focal length of the lens is $50 \mathrm{~mm}$, and the sensitivity of star magnitude is $5.5 \mathrm{Mv}$. The locations of the stars in the star images are converted to the reference star centered angular polar coordinate system and the algorithm in the paper is compared with the grid algorithm in the Cartesian coordinate system in literature [10]. The algorithm is operated in an Intel PIII-800 PC, and the statistical comparative results are shown in Table 1 below. 
Table 1. The results of the simulations

\begin{tabular}{lcc}
\hline \multicolumn{1}{c}{ Performances of the algorithm } & Algorithm in [10] & Algorithm in this paper \\
\hline Rate of successful identification & $99.36 \%$ & $99.74 \%$ \\
Mean time spent by the algorithm & $18.50 \mathrm{mS}$ & $11.40 \mathrm{mS}$ \\
Memory for star catalog & About $0.5 \mathrm{MB}$ & About $0.7 \mathrm{MB}$ \\
\hline
\end{tabular}

As demonstrated in Table 1, the algorithm presented in the paper is superior to the algorithm in the Cartesian coordinate system in both the identification rate and the time spent. The improvement in rate of success results from the consideration that the reference star may be near the brim of the FOV, as well as from the smaller radial grids adopted. The failure cases are associated with the noises inserted into the centroids of the stars.

Another improvement of the algorithm is to use the pixel-distribution of the stars in an angular polar coordinate system to coarsely identify the reference star. As the coordinates of the stars are denoted by angles, the timeconsuming trigonometric functions and vector functions are not needed to compute the inter-star angles. However, as the smaller grids are adopted, the memory required for the catalog patterns enlarges. For the radial pattern represents the angular separations between the reference star and the neighbor stars, the smaller grids are necessary to guarantee the rate of success and the robustness. As in the most patterns, there is no star in most of the radial ring strips, so most of the radial patterns include many zero bits. A data compressing method can be adopted to reduce the memory requirement for the star catalog. Nevertheless, that may somewhat delay the algorithm.

\section{Conclusions}

To speed up the star identification algorithm, an angular polar coordinate system is adopted in star sensor and a grid algorithm in the polar coordinate system is introduced. As in the angular polar coordinate system, the coordinates are all angular representations, the algorithms in the coordinate system are potential to be computationally efficient and of high identification rate. As the night sky tests and the simulations demonstrate, the algorithm proposed is excellent in both computational efficiency and identification rate. It can be used in the star sensors for high precision and high reliability in spacecraft navigation.

\section{Acknowledgements}

The work was supported by the key program of National Natural Science Foundation of China under grant No.60736010.

\section{REFERENCES}

[1] C. C. Liebe, "tar trackers for attitude determination," EEE Aerospace and Electronics Systems Magazine, Vol. 10, No. 6, pp. 10-16, June 1995.

[2] C. C. Liebe, "Accuracy performance of star tracker-a tutorial," IEEE Transactions on Aerospace and Electronic Systems, Vol. 38, No. 2, pp. 587-589, April 2002.

[3] A. Domenico, R. Giancarlo, "Brightness-independent start-up routine for star trackers," IEEE Transactions on Aerospace and Electronic Systems, Vol. 38, No. 3, pp. 813-823, July 2002.

[4] D. Mortari, M. A. Samaan, and C. Bruccoleri, et al, "The pyramid star identification technique," Journal of The Institute of Navigation, Vol. 51, No. 3, pp. 171-184, 2004.

[5] S. C. Daniel, W. P. Curtis, "Small field-of-view star identification using bayesian decision theory," IEEE Transactions on Aerospace and Electronic Systems, Vol. 36, No. 3, pp. 773-783, July 2000.

[6] M. A. Samaan, D. Mortari, and J. L. Junkins, "Non-dimensional star identification for uncalibrated star cameras," AAS/AIAA Space Flight Mechanics Meeting, Ponce, Puerto Rico, Paper No. AAS 03-131, February, 2003.

[7] M. A. Samaan, D. Mortari, and J. L. Junkins, "Recursivemode star identification algorithms," IEEE Transactions on Aerospace and Electronic Systems, Vol. 41, No. 4, pp. 1246-1254, October 2005.

[8] C. Padgett and K. Kreutz-Delgado, "A grid algorithm for autonomous star identification," IEEE Transactions on Aerospace and Electronics Systems, Vol. 33, No.1, pp. 202-213, January 1997.

[9] C. Padgett, K. Kreutz-Delgado and S. Udomkesmalee, "Evaluation of star identification techniques," Journal of Guidance, Control and Dynamics, Vol. 20, No. 2, pp. 259-267, 1997.

[10] G. Zhang, X. Wei and J. Jiang, "Full-sky autonomous star identification based on radial and cyclic features of star pattern," Image and Vision Computing, Vol. 26, No. 7, pp. 891-897, July 2008.

[11] G. B. Richard, M. G. Andrew, et al, "Advanced mathematics: precalculus with discrete mathematics and data analysis," Boston: Houghton Mifflin, 1994. 